L'imagerie aux rayons $X$ est née avec la découverte de ces ondes. Elle s'est considérablement développée grâce aux nouvelles sources de

rayonnement synchrotron, aux évolutions des détecteurs et à la révolution informatique.

Cet article présente deux

de ces développements.

Le premier est le contraste

de phase, qui permet

de visualiser des aspects

non observables autrement.

Les images de phase ont des applications dans de multiples domaines, et en particulier en paléontologie, où elles ont permis des avancées inédites. L'autre approche que nous décrivons est le couplage de l'imagerie et de la diffraction pour l'étude de matériaux poly et monocristallins, en prenant comme exemple la glace.

\section{Nouvelles applications de l'imagerie aux rayons $X$ en utilisant le rayonnement synchrotron}

José Baruchel ${ }^{(t)}\left(\right.$ baruchel@esrf.fr), Armelle Philip ${ }^{(2)}$ et Paul Tafforeau ${ }^{(1)}$

(1) Groupe Imagerie aux Rayons X, Division Expérience, ESRF, BP 220, 38043 Grenoble Cedex 9

(2) LGGE, UJF-CNRS UMR 5183, BP 96, 38402 Saint-Martin-d'Hères Cedex
Les premières images avec des rayons $\mathrm{X}$ ont été produites dès la découverte de ces ondes par Röntgen, en 1896. Le succès de la radiographie, basée sur l'absorption de ces photons (énergies $\sim 5-200 \mathrm{keV}$, et longueurs d'onde $\lambda \sim 200-5 \mathrm{pm}$ ), ne s'est pas démenti depuis lors, autant pour le médical qu'en science des matériaux. La radiographie reste très utilisée et utile : elle met en évidence les hétérogénéités associées à la variation d'absorption intégrée sur le chemin emprunté par le faisceau lors de la traversée de l'objet (fig. 1). Cette variation résulte des variations de densité ou de volume traversés, l'absorption augmentant avec le numéro atomique de l'élément présent dans l'objet. La faiblesse de l'absorption des rayons $\mathrm{X}$ par les éléments relativement légers leur permet de pénétrer des épaisseurs de matière importantes (notre corps, par exemple), et donc de scruter l'intérieur de systèmes dont la lumière visible ne permet de voir que la surface.

Le développement de l'informatique a permis l'apparition d'une version tridimensionnelle de la radiographie : la tomographie ou, en médecine, le scanner. On recombine alors numériquement de nombreuses vues bidimensionnelles, prises sous une multitude d'angles différents, pour fournir soit des rendus tridimensionnels, soit des coupes ou des projections suivant des directions arbitraires.

Par ailleurs, la diffraction des rayons $\mathrm{X}$, dont on célèbre le centenaire de la découverte, a également été utilisée pour produire des images montrant les distorsions du réseau cristallin. Ce type d'imagerie (" topographie aux rayons $\mathrm{X}$ ») échantillon

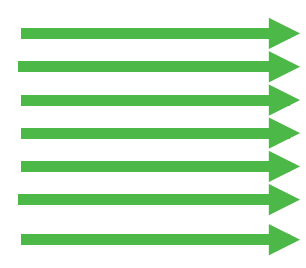

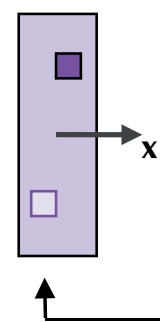

détecteur

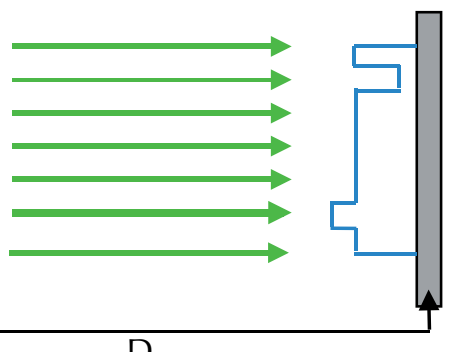

$\mathrm{D}$
1. Schéma de la radiographie. Les hétérogénéités de l'échantillon (schématisées par des carrés plus et moins absorbants que la matrice) se traduisent, au niveau de l'onde transmise, en différences d'intensité sur le détecteur (représentées par l'épaisseur des flèches dans le faisceau transmis, et par la courbe schématique montrant la variation d'intensité au niveau du détecteur) : elles résultent des variations d'absorption ou, si le faisceau est suffisamment cohérent (cas des synchrotrons modernes), de variations du front de l'onde transmise (contraste de phase). 


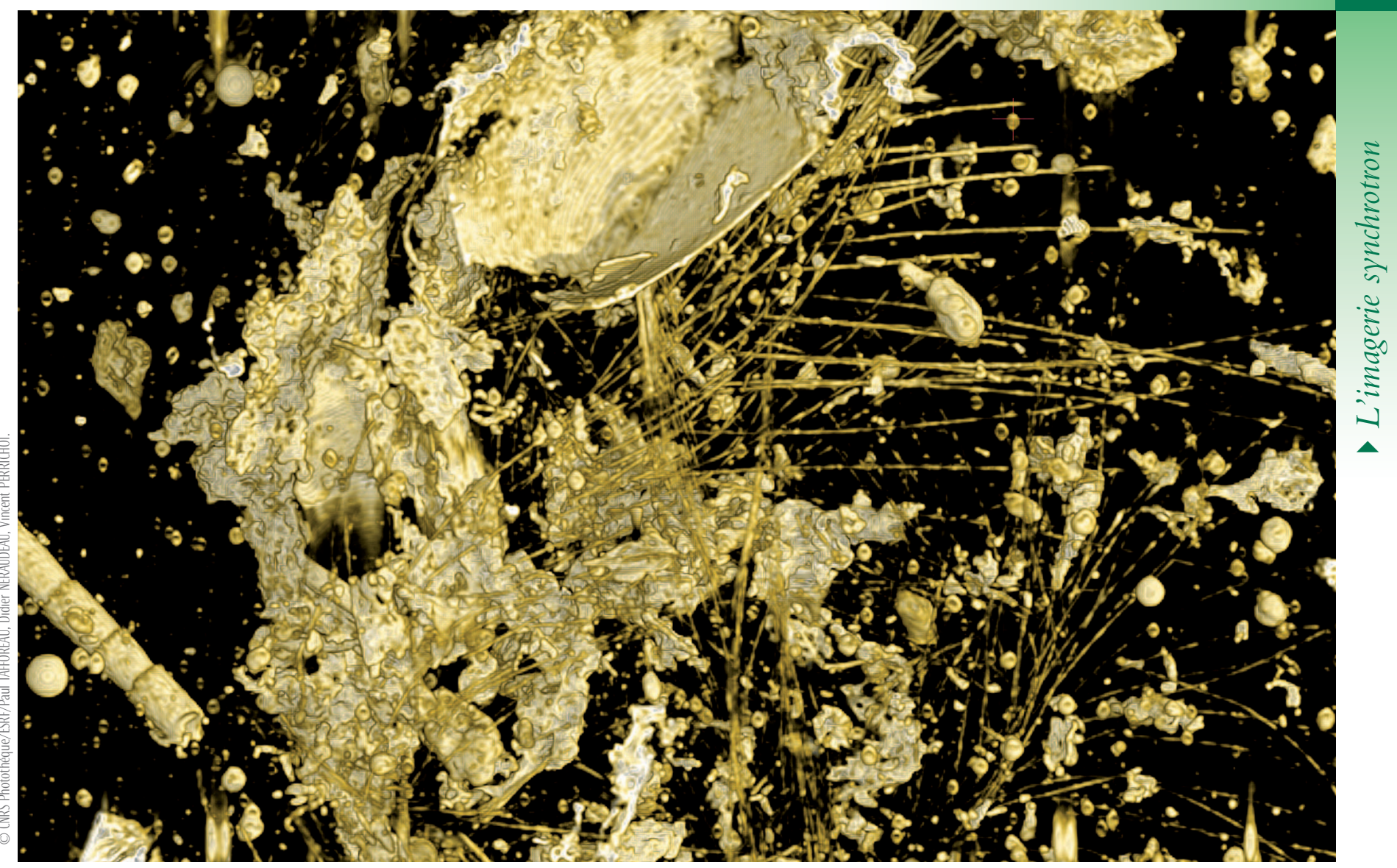

Plumes primitives d'oiseaux archaïques ou de dinosaures aviens, trouvées dans de l'ambre translucide provenant d'un gisement des Charentes, datant du Crétacé (- 100 millions d'années), imagées par microtomographie RX synchrotron à l'ESRF.

a été, en particulier, utile, dans les années 1960-1980, pour étudier les défauts dans les cristaux de silicium de haute perfection dont l'industrie microélectronique avait besoin.

La mise en service de sources de rayonnement synchrotron, dites " de $3^{\mathrm{e}}$ génération ", a permis de très nombreuses avancées, associées à la petite taille de la source et à la forte intensité des faisceaux. La petite taille angulaire de la source permet d'avoir des faisceaux " cohérents ", comme nous le verrons plus bas, et donc une approche nouvelle de la radiographie, utilisant les variations locales de phase introduites par l'objet.

Nous allons, dans cet article, présenter les avancées pour deux techniques d'imagerie aux rayons $\mathrm{X}$ : les images de phase, à haute résolution spatiale, en particulier des fossiles, qui nous serviront d'exemple, et les images en diffraction de Bragg quantitatives et tridimensionnelles, permettant de mettre en évidence les formes, orientations et distorsions des grains dans un matériau polycristallin, ou de suivre l'effet d'une contrainte appliquée dans un grain donné.
Images en contraste de phase : application aux fossiles

\section{Les bases du contraste de phase}

L'interaction des rayons $X$ et de la matière peut être décrite par un indice de réfraction complexe $n=1-\delta+i \beta$, où $\delta$ est associé à la phase $\phi=2 \pi \delta t / \lambda$, $\mathrm{t}$ étant l'épaisseur de matière traversée, et $\beta$ est proportionnel au coefficient d'absorption linéaire $\mu(\beta=\mu \lambda / 4 \pi)$. Le décrément de l'indice de réfraction $\delta$ est, dans une bonne approximation, proportionnel à la densité du matériau.

L'avantage de la «radiographie de phase " par rapport à la simple utilisation de l'absorption réside dans le gain de sensibilité qu'elle apporte, du fait des ordres de grandeur très différents de $\delta\left(\sim 10^{-5}-10^{-6}\right)$ et $\beta\left(\sim 10^{-9}-10^{-11}\right)$ dans la gamme des rayons $\mathrm{X}$. Ainsi, une variation d'épaisseur (par exemple une fissure ou une cavité dans de l'aluminium) de $5 \mu \mathrm{m}$ n'entraîne le plus souvent qu'une variation d'absorption négligeable, mais induit un déphasage de l'ordre de $\pi$.

La mise en évidence d'une variation locale de ce déphasage implique des phénomènes d'interférences. L'observation de ces interférences n'est possible, pour les ondes diffusées par des points différents d'un objet, que si l'onde incidente en ces points y a des phases bien corrélées, puisque l'interférence comme la diffraction impliquent la superposition cohérente des amplitudes de deux ou plusieurs ondes, en tenant compte de leurs phases. Ainsi, l'expérience classique des trous d'Young en optique du visible ne conduit à des interférences, donc à des franges, que si les trous sont suffisamment proches. C'est le cas, 1) si leur distance est inférieure à la longueur de cohérence $1_{c}=\lambda \alpha / 2$ où $\alpha=\mathrm{s} / \mathrm{L}$ est la taille angulaire de la source vue de l'objet, avec $\mathrm{L}$ distance source-échantillon et $\mathrm{s}$ taille de source (ici, s est la taille du faisceau d'électrons qui génère le rayonnement synchrotron), et 2) si la résolution du détecteur permet de distinguer ces franges. Ceci n'est habituellement pas le cas quand on utilise un générateur de rayons $\mathrm{X}$ pour lequel $1_{\mathrm{c}} \sim 0,2 \mu \mathrm{m}$ (avec des valeurs typiques $\mathrm{L} \sim 40 \mathrm{~cm}$ et $\mathrm{s} \sim 100 \mu \mathrm{m})$ : il n'y a pas de corrélation entre les phases de l'onde incidente, et ce sont les intensités qui s'ajoutent. En 


\section{$\gg>$}

revanche, dans les installations de rayonnement synchrotron "de $3^{\text {e généra- }}$ tion ", le faisceau émis est confiné dans un cône, autour d'une tangente à la trajectoire des électrons qui produisent les rayons $\mathrm{X}$, de largeur angulaire de l'ordre de la minute d'arc. L peut donc être grand, tout en conservant l'intensité au niveau de l'échantillon : 150 mètres pour une ligne telle que ID19 à l'ESRF, et 200 mètres pour la future ligne Nanotomography de SOLEIL. De plus, la taille de la source est petite $(\sim 30 \mu \mathrm{m})$, et donc $1_{c} \sim 200 \mu \mathrm{m}$. Ainsi des objets à absorption négligeable, ou présentant des zones de densité/absorption très proches, placés dans le trajet du faisceau, produisent des images bien contrastées, pourvu que le détecteur soit placé dans le faisceau transmis à une distance $\mathrm{D}$ (fig. 1) de l'ordre du dm [1]. Ces images montrent des systèmes de franges qui apparaissent d'autant plus que D est grand.

Le mécanisme de formation de l'image des objets de phase observés n'est autre que la diffraction de Fresnel, qui rend compte de l'effet de la propagation d'une onde sur une distance finie. Elle transforme en variations spatiales d'intensité, et donc en contraste, les hétérogénéités de la phase du faisceau de rayons $\mathrm{X}$ après traversée de l'échantillon. L'image obtenue, pour un même échantillon, dépend fortement de la distance D. Pour D petit, on observera des franges résultant des sauts de phase associés à des discontinuités, tandis que pour $\mathrm{D}$ plus grand on aura des systèmes étendus de franges d'interférence. Ces franges contiennent une information quantitative sur les déphasages. Des algorithmes pour la reconstruction de la phase ont été mis au point [2] : ils se sont révélés puissants et permettent, combinés aux procédures tomographiques, d'accéder à la répartition spatiale de l'indice de réfraction, donc de la densité. Les images de phase ont permis des avancées très significatives en science des matériaux [3], et leur usage s'est ensuite généralisé à toute une série de disciplines, qui incluent le biomédical, où ces techniques permettent de réduire la dose déposée dans les tissus biologiques.

\section{Les applications en paléontologie}

Une discipline qui a énormément profité des nouvelles possibilités offertes par la phase est la paléontologie [4]. En effet, ce type d'imagerie permet de révéler de manière non destructive des espèces fossiles inconnues dans des ambres opaques, ou de mettre en évidence des stries dentaires fournissant des informations sur l'âge et le mode de vie de l'individu étudié.

La figure 2 montre deux radiographies, obtenues à des distances $\mathrm{D}$ différentes, d'un bloc d'ambre (résine d'arbre fossile) opaque du Crétacé (il y a environ 100 millions d'années). Ces blocs d'ambre contiennent parfois des organismes fossiles sous forme d'inclusions, souvent en excellent état de conservation, que l'on trouve rarement autrement. L'ambre limpide est très connu pour ses fossiles, ainsi que pour les utilisations en joaillerie. L'imagerie synchrotron a permis la localisation et l'imagerie détaillée des inclusions dans l'ambre opaque, qui représente environ $80 \%$ de l'ambre du Crétacé, et qui était impossible à étudier jusqu'ici. Les images $2 \mathrm{a}$ et $2 \mathrm{~b}$ montrent clairement que l'absorption seule $(D \sim 5 \mathrm{~cm})$ ne permet pas de détecter les fossiles, tandis que le contraste de phase (avec D 100 cm) les montre très clairement [4]. Plus récemment, la microtomographie en contraste de phase a permis de développer des techniques de dissection virtuelle des insectes et autres organismes ayant préservé en détail leur anatomie interne [5].

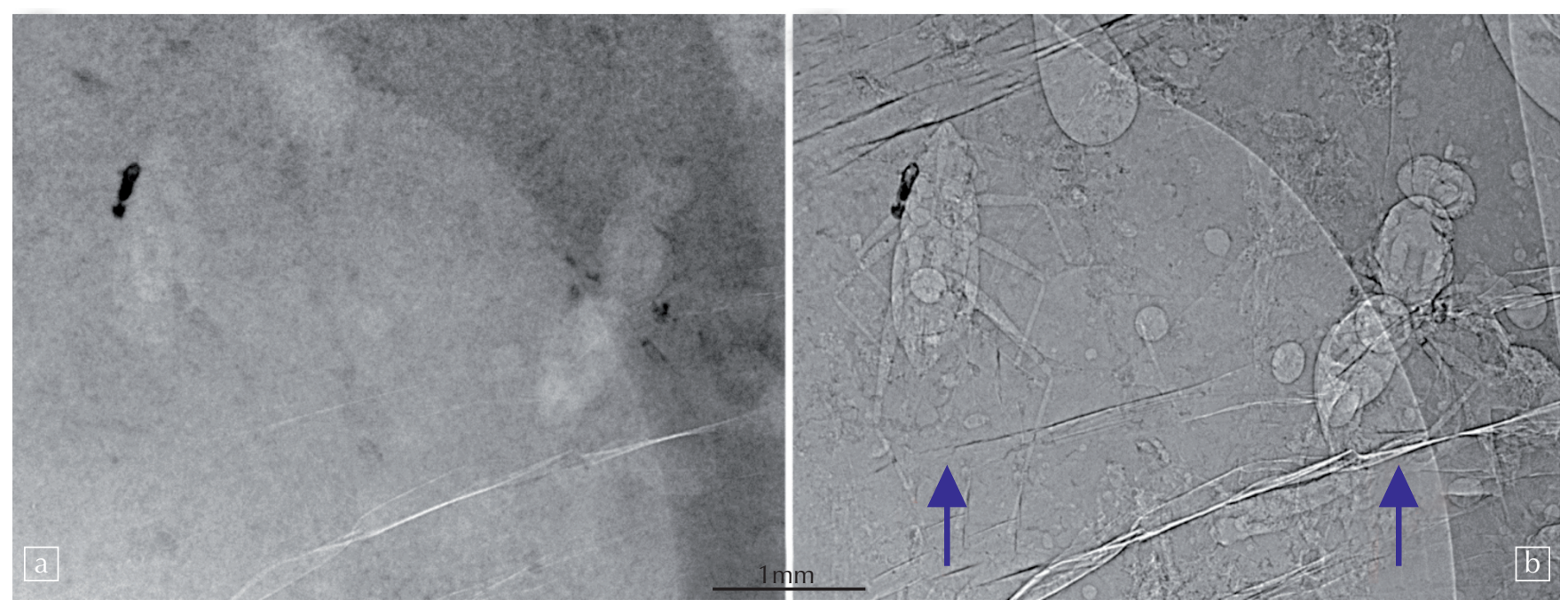

2. Deux radiographies d'un ambre opaque des Charentes. a) Absorption seule. b) Image incluant le contraste de phase, qui permet de visualiser deux insectes fossiles présents dans cet ambre (flèches bleues). 
L'étude des microstructures et structures dentaires des primates fossiles a été la première thématique paléontologique abordée grâce à l'imagerie synchrotron. Elle s'est ensuite rapidement développée et recentrée sur le développement dentaire des hominidés fossiles pour obtenir des informations sur l'évolution de ce groupe et les traits d'histoire de vie de ces formes ancestrales [6]. Les fossiles d'hominidés étant extrêmement rares, il est devenu quasiment obligatoire d'utiliser des techniques non destructives si on veut pouvoir étudier des spécimens importants. Les dents sont très contraintes génétiquement, leur morphologie est très liée au régime alimentaire, et elles enregistrent de manière détaillée leur propre développement, avec parfois des informations précises à quelques heures près. Le comptage des stries de croissance permet de calculer l'âge à la mort des juvéniles. En plus de ces différents aspects, le développement dentaire est fortement corrélé aux principaux traits d'histoire de vie des individus (naissance, développement cérébral, fin de l'enfance, maturité sexuelle, taille adulte et mort). Les dents enregistrent également les «stress » de l'individu, y compris celui de la naissance.
L'imagerie synchrotron en contraste de phase par approche multi-échelle a créé une vraie révolution en paléoanthropologie, car elle permet à présent d'accéder à la microstructure dentaire et aux stries de croissance sans devoir sectionner (et donc détruire) les spécimens.

La figure 3 montre sur des dents d'hominidés fossiles d'Afrique du Sud, datant d'environ 2 millions d'années, les différentes échelles de ce type d'étude et les microstructures dentaires associées, depuis la morphologie 3D des dents jusqu'aux stries de croissance journalière et aux prismes de l'émail, en passant par les stries de longue période et les figures associées au stress (voir légende de la figure 3).

Cette approche, en utilisant le contraste de phase, a notamment permis récemment de démontrerqueles Néanderthaliens et les hommes modernes ne se développaient pas à la même vitesse, les premiers étant environ $20 \%$ plus jeunes pour un développement similaire. Ceci constitue une information importante pour la controverse sur le statut d'espèce ou de sous-espèce de ces deux groupes.

\section{Les avancées en imagerie par diffraction de Bragg : application à la glace}

L'utilisation de la diffraction de Bragg pour former une image permet d'accéder, dans les polycristaux, à la forme et à l'orientation des grains qui les composent, et, dans un monocristal (ou pour un grain donné d'un polycristal), aux imperfections cristallines.

\section{Diffraction Contrast Tomography}

La première approche, récemment développée sous le nom de DCT ("Diffraction Contrast Tomography" [7]), combine la microtomographie avec une analyse des taches de diffraction produites par les grains monocristallins composant l'échantillon. En effet, une tomographie implique l'enregistrement de milliers d'images "radiographiques " au cours de la rotation de l'objet. Or, lors de cette rotation un grain donné se trouve, pour certains angles, en position de Bragg (c'est-à-dire vérifiant la loi de Bragg $2 \mathrm{~d} \sin \theta=\lambda$, où $\theta$ est l'angle formé par le faisceau et les plans atomiques de distance interatomique $d$ ). Dans ce cas, une partie de l'énergie est déviée de la direction incidente vers la direction diffractée : il se produit donc,

\>

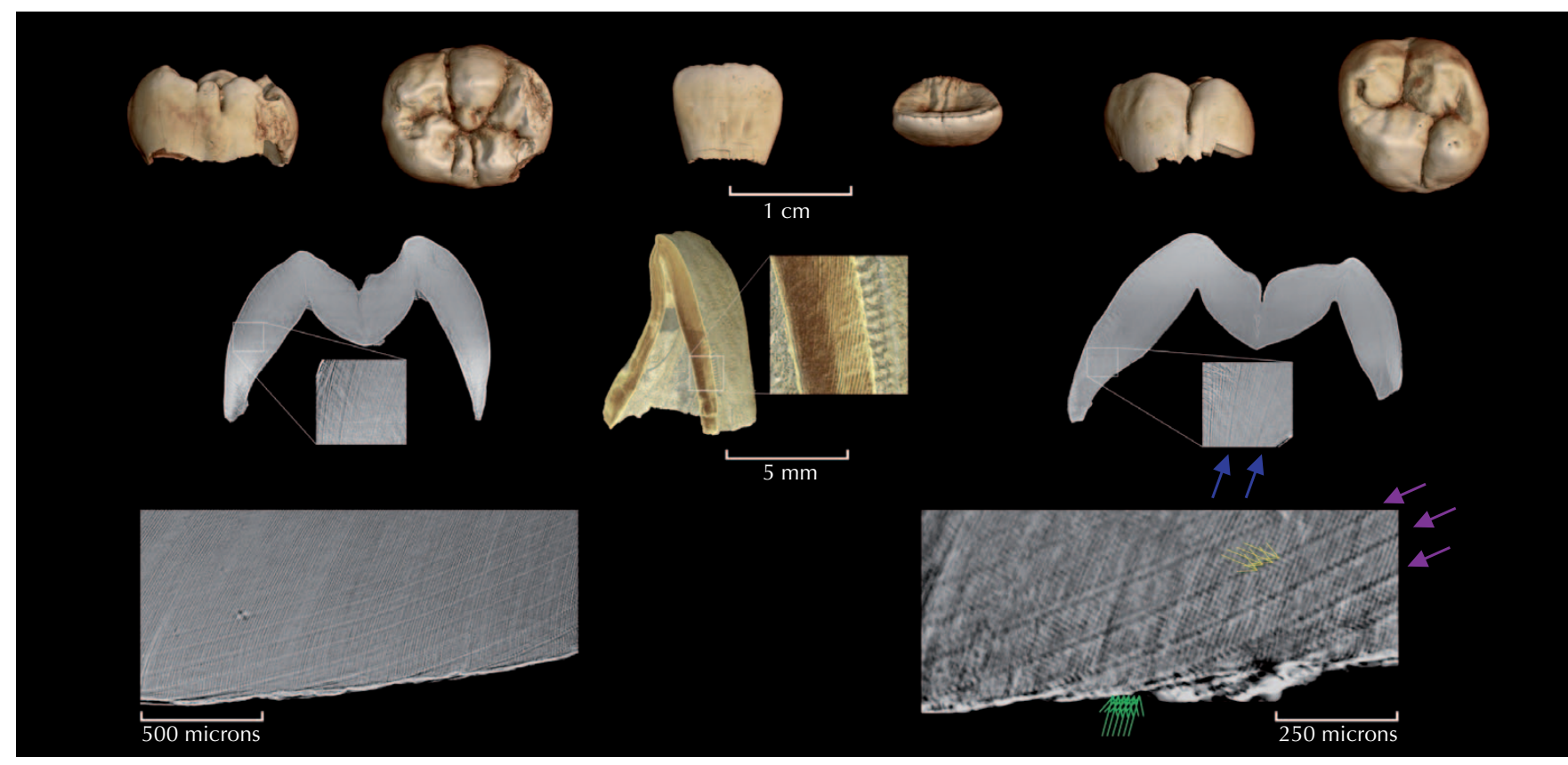

3. Images en contraste de phase de dents d'hominidés fossiles d’Afrique du Sud, âgés d'environ 2 millions d'années, montrant les différentes échelles importantes pour ce type d'étude $(\mathrm{du} \mathrm{cm}$ au $\mu \mathrm{m})$. Ligne du haut : morphologie 3D des dents. Lignes du milieu et du bas : stries de longue période ( semaine, flèches violettes), figures associées au stress (manque de nourriture, maladie..., flèches bleues), prismes de l'émail (flèches vertes) et stries de croissance journalière (flèches jaunes). Nous remercions Tanya M. Smith, Joane Pouech et Colin Mentor pour l'autorisation d'utiliser cette figure. 

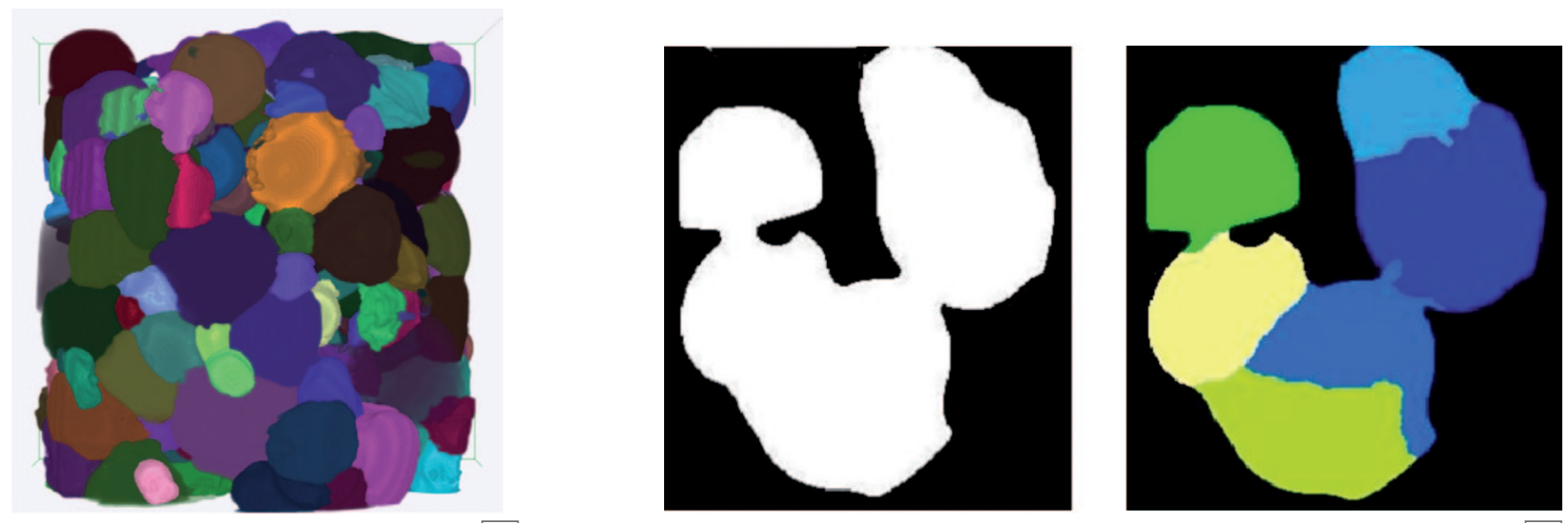

$\mathrm{b}$

4. Rendu tridimensionnel d'un échantillon de neige montrant a) les grains monocristallins de glace, et b) une coupe d'une fraction de l'image précédente en modes absorption (gauche) et diffraction (droite), avec des couleurs associées à des cristaux d'orientations différentes.

\section{\〉}

simultanément, un manque d'intensité dans l'image transmise à l'emplacement du grain en position de Bragg, et une tache diffractée que l'on enregistre également. Ceci permet, à l'aide d'algorithmes adaptés, de retrouver la forme et l'orientation cristalline des grains dans le polycristal.

Cette technique a été appliquée au cas de la neige, qui est un conglomérat de cristaux hexagonaux de glace [8]. Cette étude est importante dans le cadre d'une compréhension plus poussée de la déformation de la neige à l'échelle du grain. La figure 4 montre : a) une reconstruction tridimensionnelle de l'échantillon de neige, où chaque grain a une couleur qui correspond à son orientation de l'axe c, axe principal de la structure hexagonale, et b) la différence entre une image en absorption, qui suggère la présence de trois grains, et l'image en diffraction, qui montre la présence de six cristaux de glace d'orientations différentes dans cette zone.

\section{Rocking Curve Imaging}

La RCI ("Rocking Curve Imaging") est une autre technique d'imagerie basée sur la diffraction de Bragg, qui a été développée au synchrotron pour les monocristaux. Sur le schéma de la figure $5 \mathrm{a}$, on fait tourner le cristal, illuminé par un faisceau monochromatique, d'un petit angle $\omega$ autour d'une position de diffraction de Bragg pour une famille de plans réticulaires. Le faisceau diffracté est enregistré sur un détecteur bidimen- sionnel, comportant des "pixels ", un pixel étant associé à une zone du cristal. Sur chaque pixel, on enregistre en fonction de $\omega$ une courbe d'intensité diffractée ("rocking curve"), qui peut légèrement différer d'un pixel à l'autre, compte tenu des distorsions cristallines. Les « images » sont obtenues à partir de l'ensemble de ces enregistrements, pour plusieurs positions sur la courbe de diffraction (plusieurs valeurs de $\omega$ ). On peut extraire de l'ensemble de ces courbes de diffraction «locales" (dont chacune, mesurée sur un pixel du détecteur, est associée à une zone du cristal) plusieurs "images ». Trois de ces images (fig. 5b) sont particulièrement intéressantes : 1) celle de l'intensité intégrée, qui informe sur l'écart à la perfection cristalline ; 2) celle de la position angulaire, qui donne accès à la variation du paramètre cristallin et/ou aux courbures par rapport aux autres zones du cristal ; et 3) celle de la largeur à mi-hauteur, qui renseigne sur l'ensemble des courbures dans la zone en question. Ces trois images peuvent présenter du contraste si la distorsion locale du réseau modifie la direction ou l'intensité du faisceau diffracté. Ceci permet de visualiser et de caractériser les défauts cristallins (dislocations, inclusions...). La résolution spatiale de ces techniques est directement associée aux tailles de pixels, typiquement quelques microns.

Une extension de cette technique, dénommée "3D-RCI ", permet d'imager l'ensemble du volume du cristal [9].
La 3D-RCI a été utilisée pour suivre les premières étapes de la déformation de l'un des grains d'un tricristal de glace, produit pour cette expérience car représentant le modèle le plus simple d'un polycristal. La figure 6a est un rendu 3D de la distribution des dislocations à l'intérieur du grain (de $4 \mathrm{~mm}$ d'épaisseur, schématisé en $6 \mathrm{~b}$ ) avant déformation, à partir de la reconstruction par ordinateur des courbes de diffraction locales. La flèche violette indique la jonction triple, où les trois grains se rejoignent, qui joue un rôle primordial dans le processus de déformation.

La figure 7 montre les images après reconstruction de la largeur à mi-hauteur de ces courbes pour une tranche virtuelle de $50 \mu \mathrm{m}$ d'épaisseur située au centre de ce grain et à proximité immédiate de la jonction triple. La figure $7 \mathrm{a}$ correspond à l'état initial non déformé : les lignes verticales sont les images de dislocations individuelles, déjà observées sur la figure $6 \mathrm{a}$. Les figures $7 b$ et $7 c$ correspondent à deux états déformés après application de contraintes extérieures. En 7b, le glissement de nombreuses dislocations dans des " plans de glissement " est visible sous la forme de lignes noires verticales plus marquées que sur 7a. La figure 7c correspond à un état plus déformé, où l'on voit les prémices de la formation d'un sous-joint de grain dans le voisinage de la jonction triple. Les valeurs mesurées de la distorsion locale augmentent de deux ordres de grandeur en allant de l'état imagé en 7a à celui visualisé en $7 \mathrm{c}$. 

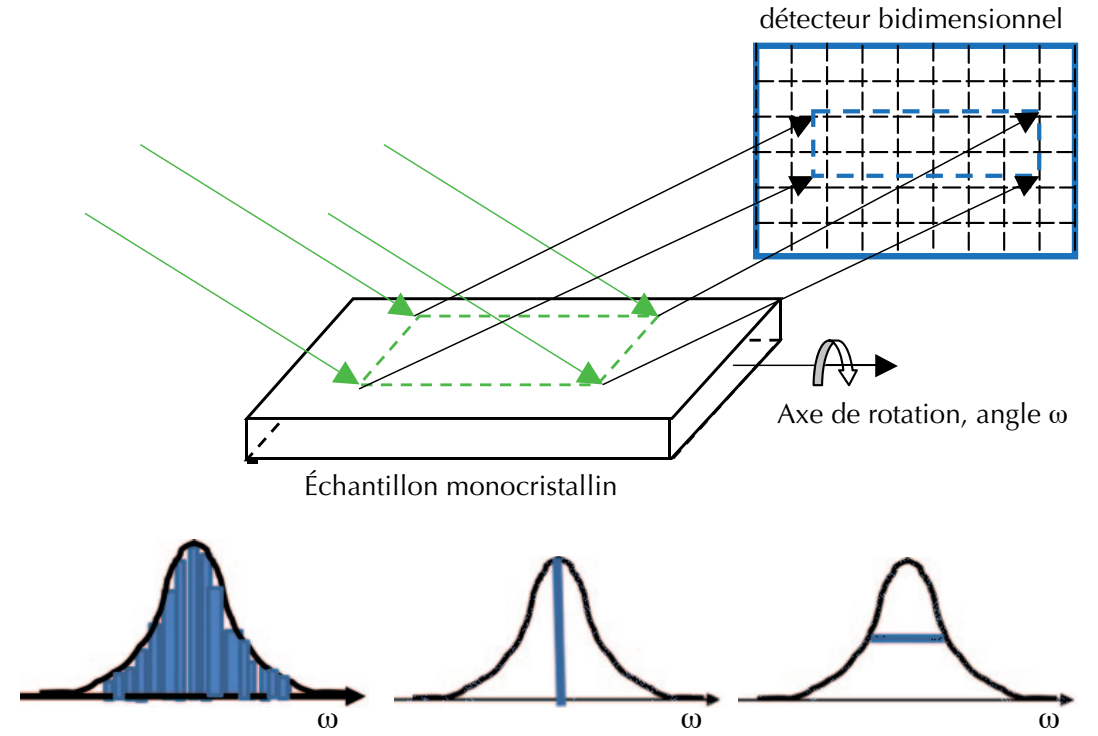

5. Schéma de la RCl ("Rocking Curve Imaging").

(a) Le faisceau monochromatique incident est diffracté par l'échantillon monocristallin en position de Bragg. Cet échantillon subit une petite rotation d'angle $\omega$ autour de la position de Bragg, qui permet d'enregistrer les courbes de diffraction « locales » pour chaque pixel du détecteur bidimensionnel.

(b) Représentation schématique de la courbe de diffraction enregistrée sur un pixel, en fonction de l'angle $\omega$, pour montrer les trois quantités que l'on extrait habituellement : l'intensité intégrée (surface sous la courbe de diffraction), la position angulaire du centre de gravité de la courbe et sa largeur à mi-hauteur.

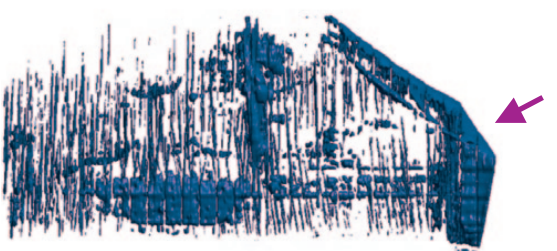

a

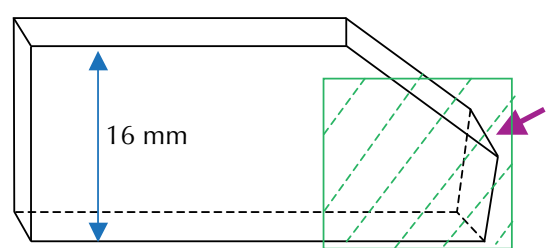

b
6. Rendu tridimensionnel d'un grain d'un tricristal de glace avant déformation. a) Seules les zones présentant une " distorsion " plus grande que deux fois celles du cristal " parfait " sont représentées : les lignes verticales correspondent à des dislocations individuelles ; les taches étendues, à des défauts de surface (au centre) ou, à droite, aux zones de parois de joints de grains avec, en particulier, la jonction triple où les trois grains différents se rejoignent (flèche violette). b) Schéma du grain imagé en 6a ; le rectangle hachuré en vert correspond à la zone représentée en figure 7.
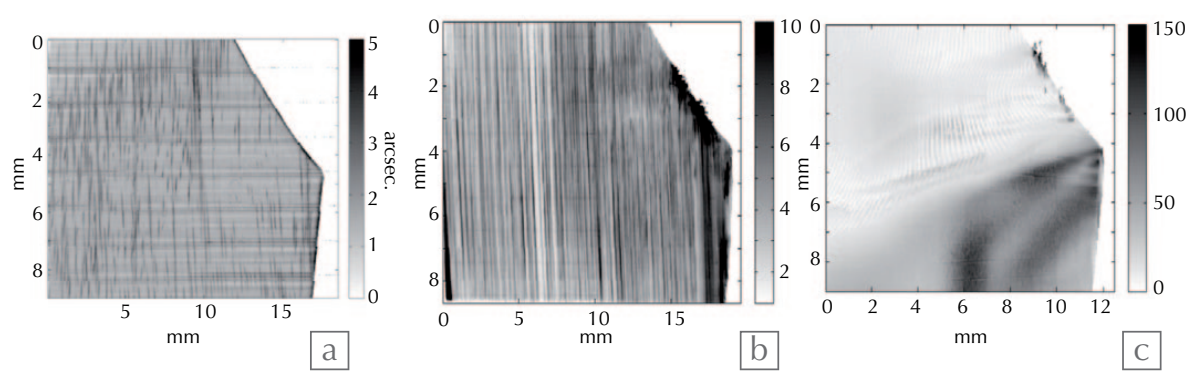

7. Images reconstruites de la distribution des largeurs à mi-hauteur des courbes de diffraction, dans une tranche virtuelle centrale de $50 \mu \mathrm{m}$ du grain de tricristal de glace de $4 \mathrm{~mm}$ d'épaisseur, pour la zone indiquée par un rectangle vert sur la figure 6b. Les échelles sont données sur le côté des images, en secondes d'arc. Les images (a), (b) et (c) correspondent à des étapes de déformation successives : (a) cristal non déformé $\left(\sim 10^{2} \mathrm{~cm} / \mathrm{cm}^{3}\right.$ dislocations - lignes verticales -$)$; (b) après application d'une contrainte externe, montrant des bandes de glissement ; (c) état plus déformé, avec l'amorce d'un sous-joint de grain dans le voisinage de la jonction triple (voir texte).

\section{Conclusion}

L'imagerie aux rayons X utilisant le rayonnement synchrotron « de $3^{\text {e }}$ génération" a progressé de manière remarquable au cours des dernières années. Les techniques d'imagerie constituent l'une des priorités des plans de jouvence et de rénovation de plusieurs de ces grands instruments : elles sont en effet associées à de nombreux résultats scientifiques dans des domaines très variés, qui vont de la science des matériaux au patrimoine, en passant par les objets d'intérêt biologique.

Les nouvelles sources de rayonnement synchrotron permettent d'exploiter, dans un mode imagerie, une grande variété d'interactions entre les rayons $\mathrm{X}$ et la matière. Les techniques d'imagerie de phase se développent constamment, dans de nombreuses directions. De multiples travaux ont utilisé les diagrammes produits en régime de Fraunhofer (diagrammes «à l'infini »), en appliquant des algorithmes sophistiqués pour la reconstitution de la phase. De nombreuses techniques d'imagerie en diffraction, dont la DCT ou la 3D-RCI ne sont que des exemples, offrent de nouvelles possibilités pour l'étude des matériaux. Le balayage avec un nanofaisceau $(\sim 50 \mathrm{~nm})$ fournit des cartes de la fluorescence d'un petit volume dans l'échantillon. Elles permettent d'obtenir une "image " tridimensionnelle de la composition chimique (en particulier, des éléments traces, qui souvent jouent un rôle important), offrant ainsi des possibilités accrues tant pour l'étude de nanomatériaux que pour celle de l'intérieur des cellules biologiques.

\section{Références}

1• A. Snigirev et al., Rev. Sci. Instrum. 66 (1995) 5486 P. Cloetens et al., J. Phys. D: Appl.Phys. 29 (1996) 133.

2• P. Cloetens et al., Appl. Phys. Lett., 75 (1999) 2912 2914 ; D.M. Paganin, Coherent X-ray Optics, Oxford Univ. Press (2006)

$3 \bullet X$ ray Tomography in Material Science, ed. par J. Baruchel et al., HERMES, Paris (2000).

4• P. Tafforeau et al., Appl. Phys. A 83 (2006) 195.

5• M. Perreau et al., Systematic Entomology 36 (2011) 573.

6• P. Tafforeau et al., Journal of Human Evolution 54 (2008) 272 ; T.M. Smith et al., PNAS 107 (2010) 20923.

7• A. King et al., Science 321 (2008) 382.

8• S. Rolland du Roscoat et al., Advanced Engineering Materials 13 (2011) 128

9• R. T. Kluender, Thèse de Doctorat, Université de Grenoble (29 septembre 2011). 